\title{
Phase control of spatial interference from two duplicated two-level atoms
}

\author{
Luling Jin, ${ }^{1}$ Yueping Niu, ${ }^{1, *}$ and Shangqing Gong ${ }^{1, \oplus}$ \\ ${ }^{1}$ State Key Laboratory of High Field Laser Physics, \\ Shanghai Institute of Optics and Fine Mechanics, \\ Chinese Academy of Sciences, Shanghai 201800, China
}

(Dated: October 31, 2018)

\begin{abstract}
We report the phase control of spatial interference of resonance fluorescence from two duplicated two-level atoms, driving by two orthogonally polarized fields. In this closed-loop system, the relative phase is of crucial importance to the recovery of the interference patten in the fluorescence light even with strong driving. In order to improve the experimental realizability, we propose a scheme to recover the visibility with fixed relative phase by adjusting the relative intensities between the two driving fields or alternatively by using a standing-wave field.
\end{abstract}

PACS numbers:

Young's double-slit experiment is important to our understanding of the wave nature of light, which exhibits the first-order coherence properties of light [1]. Recently, there has been considerable interest in the interference of the fluorescence light from two driven atoms which play the role of the slits in Young's experiment [2 6]. Remarkably, Eichmann et al. carried out a very nice experiment where the two slits were replaced by two ${ }^{198} \mathrm{Hg}^{+}$ions in a trap and observed the interference patten in the light scattered from the two ions [2]. However, it has been shown that, in the strong field limit, the two-particle collective dressed states are uniformly populated, so that the interference vanishes at strong driving [3-6]. This restricts potential applications, e.g., in coherent backscattering from disordered structures of atoms [7], the generation of squeezed coherent light by scattering light off of a regular structure [8], the lithography [9], or precision measurements and optical information processing.

Macovei et al investigated the radiation from a collective of atoms [10], and very recently, they proposed a scheme to recover first-order interference with almost full visibility in strong fields, by tailoring the surrounding electromagnetic bath with a suitable frequency dependence, e.g., with the help of cavities [11]. In the modified reservoirs, the population in the collective many-particle dressed states was repopulated, so that the possible scattering pathways was modified and resulted in the recovery of the interference.

In this Letter, we propose a different scheme to recover the spatial interference of resonance fluorescence from two duplicated two-level atoms via controlling the relative phase of driving fields. The atomic system, investigated before by Bouchene and coworkers [12], is driven by two orthogonally polarized fields, and thus a closedloop system is formed, so that the relative phase becomes very important and can be used to control the optical property of the medium. We find that if an appropriate

*Electronic address: niuyp@mail.siom.ac.cn

${ }^{\dagger}$ Electronic address: sqgong@mail.siom.ac.cn relative phase is chosen, the interference patten could be recovered even in strong driving fields, by adjusting the relative intensities between the two driving fields. Based on the technology of phase control [13], this scheme may provide experimental maneuverability. Later, we replace one driving field with a standing-wave field, and find that the restriction on the phase shift can be overcome by adjusting the distance between the atoms and the screen.

The atoms used here are modeled as duplicated twolevel atoms [see Fig. 1(a)]. We consider the $F=1 / 2 \leftrightarrow$ $F=1 / 2$ transition (energy $\hbar \omega_{0}$ ) excited by orthogonally polarized fields. The system could be realized in ${ }^{6} \mathrm{Li}$ atom. The two lower (upper) states $\{|1\rangle,|2\rangle\}(\{|3\rangle,|4\rangle\}$ ) with energies $E_{1}=E_{2}\left(E_{3}=E_{4}\right)$ correspond to the degenerate states of the lever ${ }^{2} S_{1 / 2} F=1 / 2\left({ }^{2} P_{1 / 2} F=\right.$ $1 / 2)$ with $m_{F}= \pm 1 / 2$. The transitions with identical $m_{F}$ (the transitions $|1\rangle \leftrightarrow|3\rangle$ and $|2\rangle \leftrightarrow|4\rangle$ ) are coupled by the $\pi$-polarized field, while the transitions with different $m_{F}(|2\rangle \leftrightarrow|3\rangle$ and $|1\rangle \leftrightarrow|4\rangle)$ are coupled by $\sigma$-polarized field. Thus, a closed-loop system is formed, and it allows us to control optical properties of the medium by the phases of the laser fields. The electric fields, having the same frequency $\omega$, are $\vec{E}_{\pi}(y, t)=\vec{e}_{z} \epsilon_{\pi}(y) e^{-i(\omega t-k y)}+$ c.c. and $\vec{E}_{\sigma}(z, t)=\vec{e}_{x} \epsilon_{\sigma}(z) e^{-i(\omega t-k y)} e^{-i \phi}+$ c.c. , where $\epsilon_{i}$ is the amplitude with $i \in\{\sigma, \pi\}, \omega$ is the frequency, $k$ is the wave vector, and $\phi$ is the relative phase between these driving fields. We assume that both excited states have the same decay rate $\gamma$ to the lower levels.

The atomic dipole operator is the sum of atomic raising $\mu^{\uparrow}$ and lowering $\mu^{\downarrow}$ operators whose compnents are [14]

$$
\begin{array}{r}
\mu_{x}^{\downarrow}=\mu(|1\rangle\langle 4|+| 2\rangle\langle 3|) \hat{x}, \\
\mu_{y}^{\downarrow}=-i \mu(|1\rangle\langle 4|-| 2\rangle\langle 3|) \hat{y}, \\
\mu_{z}^{\downarrow}=\mu(|2\rangle\langle 4|-| 1\rangle\langle 3|) \hat{z},
\end{array}
$$

where $\mu_{k}^{\downarrow}$ is the $k$ component of the atomic dipole, $\mu$ is the dipole matrix element, and $\hat{x}, \hat{y}$, and $\hat{z}$ are the usual Cartesian unit vectors. In the interaction picture, the Hamiltonian of the system in an appropriate roating 


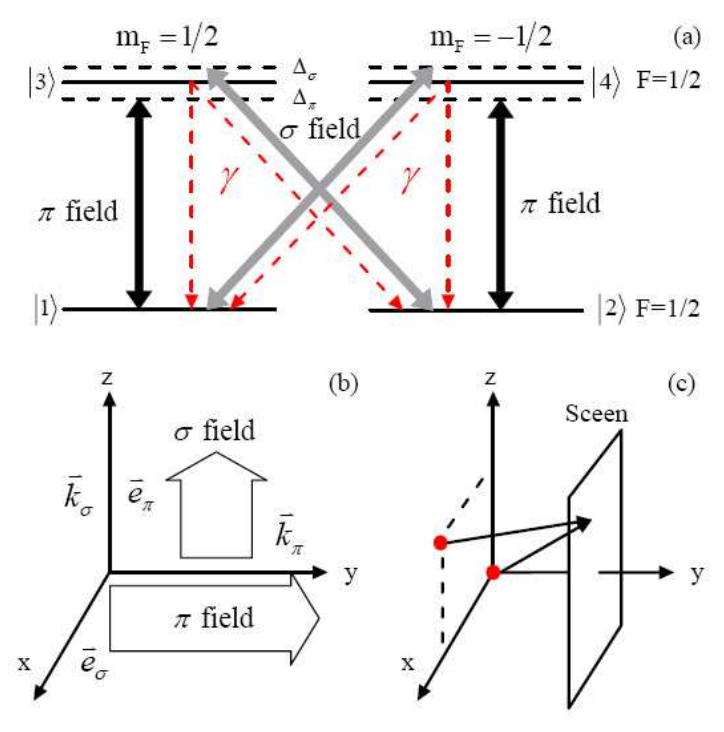

FIG. 1: (Color online) (a) Energy level structure for consideration. The transitions with identical $m_{F}(|1\rangle \leftrightarrow|3\rangle$ and $|2\rangle \leftrightarrow|4\rangle)$ are coupled by the $\pi$-polarized field, while the transitions with different $m_{F}(|2\rangle \leftrightarrow|3\rangle$ and $|1\rangle \leftrightarrow|4\rangle)$ are coupled by $\sigma$-polarized field. (b) Fields configurations. (c) Schematic diagram of the setup.

frame can be written as

$$
\mathrm{H}=\hbar\left(\begin{array}{cccc}
0 & 0 & \Omega_{\pi} & -\Omega_{\sigma} e^{-i \phi} \\
0 & 0 & -\Omega_{\sigma}^{*} e^{-i \phi} & -\Omega_{\pi} \\
\Omega_{\pi} & -\Omega_{\sigma} e^{i \phi} & \Delta & 0 \\
-\Omega_{\sigma}^{*} e^{i \phi} & -\Omega_{\pi} & 0 & \Delta
\end{array}\right),
$$

where $\Delta=\omega_{0}-\omega$ is the detuning, and the Rabi frequencies are defined as $\Omega_{\pi}=\mu \epsilon_{\pi} / 2 \hbar$ and $\Omega_{\sigma}=\mu \epsilon_{\sigma} / 2 \hbar$. The dynamics of the system can be described using densitymatrix approach as

$$
\dot{\rho}=-\frac{i}{\hbar}[\mathrm{H}, \rho]+L[\rho(t)]
$$

The Liouvillian matrix $L[\rho(t)]$, which describes relaxation by spontaneous decay, is given by

$L[\rho(t)]=\left(\begin{array}{cccc}\gamma\left(\rho_{33}+\rho_{44}\right) & 0 & -\gamma \rho_{13} & -\gamma \rho_{14} \\ 0 & \gamma\left(\rho_{33}+\rho_{44}\right) & -\gamma \rho_{23} & -\gamma \rho_{24} \\ -\gamma \rho_{31} & -\gamma \rho_{32} & -2 \gamma \rho_{33} & -2 \gamma \rho_{34} \\ -\gamma \rho_{41} & -\gamma \rho_{42} & -2 \gamma \rho_{43} & -2 \gamma \rho_{44}\end{array}\right)$

We define $n_{g}=\rho_{11}+\rho_{22}$ and $n_{e}=\rho_{33}+\rho_{44}$ as the ground and excited populations, and the coherences $\rho_{\pi}=\rho_{42}-\rho_{31}, \rho_{\sigma}=\rho_{32}+\rho_{41}$ responsible for the $\pi$ - and $\sigma$-polarized radiated fields, respectively. We solve the density-matrix equation (3) in steady state while considering the situation that both driving fields are exactly resonant with corresponding transitions $(\Delta=0)$ and all parameters are dimensionless and normalized by $\gamma$ and we have

$$
\begin{aligned}
& n_{e}=\frac{1}{2}\left[1-\frac{\Omega_{\sigma}^{2}+\Omega_{\pi}^{2}}{\Omega_{\sigma}^{2}+\Omega_{\pi}^{2}+2\left(\Omega_{\sigma}^{2}-\Omega_{\pi}^{2}\right)^{2}+4 \Omega_{\sigma}^{2} \Omega_{\pi}^{2}(\cos 2 \phi+1)}\right], \\
& \rho_{\sigma}=\frac{\Omega_{\sigma}\left[\left(\Omega_{\pi}^{2}-\Omega_{\sigma}^{2}\right) \sin \phi+i\left(\Omega_{\pi}^{2}+\Omega_{\sigma}^{2}\right) \cos \phi\right]}{\Omega_{\sigma}^{2}+\Omega_{\pi}^{2}+2\left(\Omega_{\sigma}^{2}-\Omega_{\pi}^{2}\right)^{2}+4 \Omega_{\sigma}^{2} \Omega_{\pi}^{2}(\cos 2 \phi+1)}, \\
& \rho_{\pi}=\frac{\Omega_{\pi}\left[i\left(\Omega_{\pi}^{2}+\Omega_{\sigma}^{2} \cos 2 \phi\right)-\Omega_{\sigma}^{2} \sin 2 \phi\right]}{\Omega_{\sigma}^{2}+\Omega_{\pi}^{2}+2\left(\Omega_{\sigma}^{2}-\Omega_{\pi}^{2}\right)^{2}+4 \Omega_{\sigma}^{2} \Omega_{\pi}^{2}(\cos 2 \phi+1)}
\end{aligned}
$$

From the population and the coherences in the steady state, we find that the relative phase plays an important role in the atomic dynamics and should effect the interference patten.

What we are interested in is the far-field interference pattern from two duplicated two-level atoms. We have calculated the steady-state solutions for the atomic coherences and populations for the case of a single atom that interacts with two classical laser light field linearly polarized along the $x$ axis and $z$ axis, respectively. Now in our calculation of the far-field interference pattern, we consider that the separation between the atoms is large enough that they may be treated independently. The observing screen is placed in the far-field (large $y$ ) and oriented in the $x-z$ plane, illustrated in Fig. 1(c). At a point $\left(\tau_{1}, \tau_{2}\right)$ on the screen (where $\tau_{i}$ is the light travel time from the $i$ th atom to the observation point, $i=1,2)$, the intensity of the light is

$$
I\left(\tau_{1}, \tau_{2}\right) \propto\left\langle E_{x}^{\uparrow} E_{x}^{\downarrow}+E_{z}^{\uparrow} E_{z}^{\downarrow}\right\rangle
$$

where

$$
E_{k}^{\uparrow}\left(t ; \tau_{1}, \tau_{2}\right) \propto e^{-i \omega\left(t-\tau_{1}\right)} u_{k}^{\uparrow}+e^{-i \omega\left(t-\tau_{2}\right)} \mathcal{U}_{k}^{\uparrow}
$$

- for $k \in x, z, u$ and $\mathcal{U}$ are the atomic dipoles of the first and second atoms respectively and $\omega$ is the angular frequency of the laser light. Since the atoms can be considered as independent and identical, the intensity of the interference pattern when all the light is detected is given by

$$
\begin{aligned}
I\left(\tau_{1}, \tau_{2}\right) & \propto\left\langle u_{x}^{\uparrow} u_{x}^{\downarrow}+\mathcal{U}_{x}^{\uparrow} \mathcal{U}_{x}^{\downarrow}+u_{z}^{\uparrow} u_{z}^{\downarrow}+\mathcal{U}_{z}^{\uparrow} \mathcal{U}_{z}^{\downarrow}\right\rangle \\
& +\left\langle u_{x}^{\uparrow} \mathcal{U}_{x}^{\downarrow}\right\rangle e^{i \omega\left(\tau_{1}-\tau_{2}\right)}+\left\langle u_{x}^{\downarrow} \mathcal{U}_{x}^{\uparrow}\right\rangle e^{-i \omega\left(\tau_{1}-\tau_{2}\right)} \\
& +\left\langle u_{z}^{\uparrow} \mathcal{U}_{z}^{\downarrow}\right\rangle e^{i \omega\left(\tau_{1}-\tau_{2}\right)}+\left\langle u_{z}^{\downarrow} \mathcal{U}_{z}^{\uparrow}\right\rangle e^{-i \omega\left(\tau_{1}-\tau_{2}\right)}
\end{aligned}
$$


Each component in Eq. 8 in the steady state is

$$
\begin{aligned}
\left\langle u_{x}^{\uparrow} u_{x}^{\downarrow}\right\rangle_{s s} & =\left\langle\mathcal{U}_{x}^{\uparrow} \mathcal{U}_{x}^{\downarrow}\right\rangle_{s s} \\
& \propto \mu^{2}\langle(|4\rangle\langle 1|+| 3\rangle\langle 2|)(|1\rangle\langle 4|+| 2\rangle\langle 3|)\rangle_{s s} \\
& =\mu^{2}\langle\mid 4\rangle\langle 4|+| 3\rangle\langle 3 \mid\rangle_{s s} \\
& =\mu^{2} n_{e}, \\
\left\langle u_{z}^{\uparrow} u_{z}^{\downarrow}\right\rangle_{s s} & =\left\langle\mathcal{U}_{z}^{\uparrow} \mathcal{U}_{z}^{\downarrow}\right\rangle_{s s} \\
& \propto \mu^{2}\langle(|4\rangle\langle 2|-| 3\rangle\langle 1|)(|2\rangle\langle 4|-| 1\rangle\langle 3|)\rangle_{s s} \\
& =\mu^{2} n_{e}, \\
\left\langle u_{x}^{\uparrow} \mathcal{U}_{x}^{\downarrow}\right\rangle_{s s} & =\left\langle u_{x}^{\uparrow} \mathcal{U}_{x}^{\uparrow}\right\rangle_{s s}^{*} \\
& \propto \mu^{2}\left\langle u_{x}^{\uparrow}\right\rangle_{s s}\left\langle\mathcal{U}_{x}^{\downarrow}\right\rangle_{s s} \\
& =\mu^{2}\langle(|4\rangle\langle 1|+| 3\rangle\langle 2|)\rangle_{s s}\langle(|1\rangle\langle 4|+| 2\rangle\langle 3|)\rangle_{s s} \\
& =\mu^{2} \rho_{\sigma} \rho_{\sigma}^{*}, \\
\left\langle u_{z}^{\uparrow} \mathcal{U}_{z}^{\downarrow}\right\rangle_{s s} & =\left\langle u_{z}^{\uparrow} \mathcal{U}_{z}^{\uparrow}\right\rangle_{s s}^{*} \\
& \propto \mu^{2}\left\langle u_{z}^{\uparrow}\right\rangle_{s s}\left\langle\mathcal{U}_{z}^{\downarrow}\right\rangle_{s s} \\
& =\mu^{2}\langle(|4\rangle\langle 2|-| 3\rangle\langle 1|)\rangle_{s s}\langle(|2\rangle\langle 4|-| 1\rangle\langle 3|)\rangle_{s s} \\
& =\mu^{2} \rho_{\pi} \rho_{\pi}^{*} .
\end{aligned}
$$

Unlike the results in Refs. [5, [6], the cross term $\left\langle u_{x}^{\uparrow} \mathcal{U}_{x}^{\downarrow}\right\rangle_{s s}$ and $\left\langle\mathcal{U}_{x}^{\uparrow} u_{x}^{\downarrow}\right\rangle_{s s}$ contribute to the total intensity due to the driving of the $\sigma$-polarized field that $\left|\rho_{\sigma}\right| \neq 0$. Thus the intensity in Eq. (8) is

$I\left(\tau_{1}, \tau_{2}\right) \propto 4 n_{e}\left\{1+\frac{1}{2 n_{e}}\left(\rho_{\sigma} \rho_{\sigma}^{*}+\rho_{\pi} \rho_{\pi}^{*}\right) \cos \left[\omega\left(\tau_{1}-\tau_{2}\right)\right]\right\}$,

The visibility of the interference patten is defined as $V=\left(I_{\max }-I_{\min }\right) /\left(I_{\max }+I_{\min }\right)$. In our duplicated two-level atomic system, the visibility can be calculated by using the steady states solutions (Eq. (5)

$$
\begin{aligned}
V & =\frac{1}{2 n_{e}}\left(\rho_{\sigma} \rho_{\sigma}^{*}+\rho_{\pi} \rho_{\pi}^{*}\right) \\
& =\frac{1}{2} \frac{\Omega_{\sigma}^{2}+\Omega_{\pi}^{2}}{\Omega_{\sigma}^{2}+\Omega_{\pi}^{2}+2\left(\Omega_{\sigma}^{2}-\Omega_{\pi}^{2}\right)^{2}+4 \Omega_{\sigma}^{2} \Omega_{\pi}^{2}(\cos 2 \phi+1)} .
\end{aligned}
$$

Compared with Eq. [5(a), it is easy to see that

$$
V+n_{e}=1 / 2 .
$$

We noticed that both of the two components polarized in $x$ and $z$ axis contribute to the total intensity detected on the screen. In this duplicated two-level atomic system, when the $\pi$ - and $\sigma$-polarized fields are applied simultaneously, the two components could not be separated and the visibility is always less than one-half.

From Eq.11, we can see that the interference patten of the resonance fluorescence from two duplicated two-level atoms is related to the Rabi frequencies of the driving fields, and especially to the relative phase $\phi . V$ as the function of the relative phase between these two driving fields reaches its maximum $V_{\max }$ when $\cos 2 \phi=-1$ ( $\phi=$ $\frac{\pi}{2} \pm 2 n \pi, n$ is an arbitrary integer):

$$
V_{\max }=\frac{1}{2} \frac{\Omega_{\sigma}^{2}+\Omega_{\pi}^{2}}{\Omega_{\sigma}^{2}+\Omega_{\pi}^{2}+2\left(\Omega_{\sigma}^{2}-\Omega_{\pi}^{2}\right)^{2}} .
$$

From Eq. 5(a), in this case, the excited population reaches the minimum

$$
n_{\text {emin }}=\frac{1}{2}\left[1-\frac{\Omega_{\sigma}^{2}+\Omega_{\pi}^{2}}{\Omega_{\sigma}^{2}+\Omega_{\pi}^{2}+2\left(\Omega_{\sigma}^{2}-\Omega_{\pi}^{2}\right)^{2}}\right] .
$$

It has been confirmed that in strong driving situation, the interference patten vanishes [3-6]. From our main results Eqs. 11 to 14, we find that in our scheme, the visibility could be realized even in the strong driving situation due to the relative phase $\phi$. Figure 2 shows how the visibility evolves under different driving situations. Without the phase difference, the visibility will fall towards zero rapidly as the driving field strengths are increased [see Fig. 2(a)]. When the phase difference between the two driving fields $\phi$ is taken into consideration, phase dependent interference of resonance fluorescence will show up. As we have analyzed from the expression of $V$, when $\phi=\pi / 2$, the visibility will reach its maximum. From Figs. 2(b) and (c) we find that even driven by strong fields, the interference patten could still be observed when the relative phase is chosen to be around $\pi / 2$. The relative phase is the determinant in the recovery of the interference patten. Figure 2 (d) shows $V$ vs $\phi$, when the Rabi frequencies of the two fields are $\Omega_{\sigma}=\Omega_{\pi}=1,5$ and 10 as examples. When the relative phase $\phi$ is exactly equal to $\pi / 2$, we find that $V=1 / 2$, and from the Eq. 12, there is no population on the excited states, that is to say, no fluorescence could be detected. This can be explained from further investigations on Eqs. 13 and 14. If $\Omega_{\sigma}=\Omega_{\pi}$, the extremum of $V$ and $n_{e}$ goes to $V_{\max }=1 / 2$ and $n_{\text {emin }}=0$ when $\phi=\pi / 2$.

In order to remove the restriction that $\phi$ can not be exactly $\pi / 2$ in experiments when the driving fields have the same intensities, we investigate the influence of the strengths of those two driving fields on the interference, especially when $\phi=\pi / 2$. We define the ratio of the Rabi frequencies $r=\Omega_{\pi} / \Omega_{\sigma}$. It is shown in Fig. 3(a) that a peak emerges when $r=1$. As the strengths of the driving fields increase, the peak becomes narrower. This, however, provides the feasibility to recover the interference under strong driving with $\phi=\pi / 2$, by choosing the ratio between the two driving fields properly. We choose $r=0.9,0.95$ and 0.99 for examples in Fig. 3(b). It is shown that as $r$ gets closer to 1 , the visibility could survive even when driven by strong fields. Thus, by adjusting the relative intensities of driving fields, the patten would reappearance under strong driving even when $\phi=\pi / 2$.

We notice that the adjusting of the relative intensities works only when $r$ is modified around one. It is known that a standing-wave field is periodic in space and oscillates between its minimum and maximum. An idea 

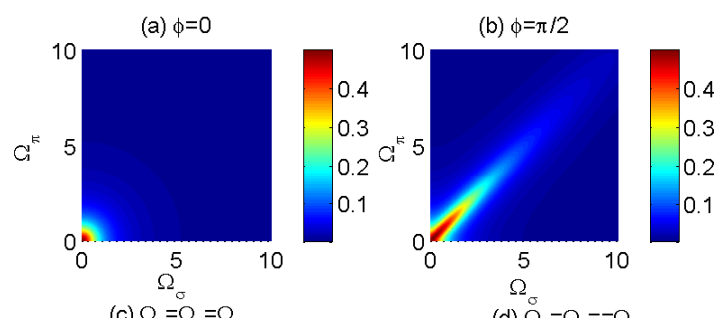

(c) $\Omega_{\sigma}=\Omega_{\pi}=\Omega$

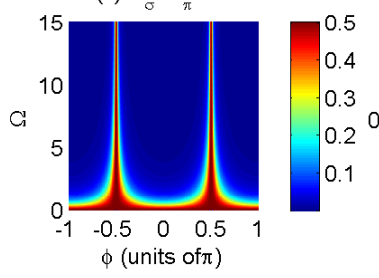

(d) $\Omega_{\sigma}^{\sigma}=\Omega_{\pi}=\Omega$

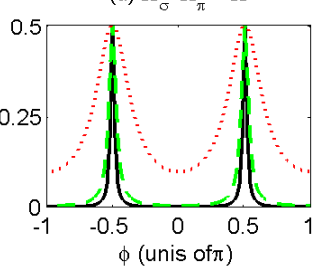

FIG. 2: (Color online) The visibility $V$ in different driving situations: (a) the relative phase is zero, (b) the relative phase is $\pi / 2$, and (c) the intensities of the driving fields are equal. (d) Some examples in (c). The dotted (red) curve: $\Omega=1$, the dashed (green) curve: $\Omega=5$, and the solid (black) curve: $\Omega=10$.

came into our mind that we can replace one of the driving field with a standing-wave field. As interference patten is observed in the $x-z$ plane, we then use a $\pi$-polarized standing-wave field, which is applied along the $y$ axis and therefore $\Omega_{\pi}(y)=\Omega \sin (k y)$. The observing screen is fixed at the end of the cavity, and the atoms are located in $x-z$ plane so that they experience the same driving fields. As the intensity of the standing wave is positiondependent, the interference patten in $x-z$ plane is related to the detected distance between the screen to the plane the atoms are located. In order to compare with the above work, we choose $\Omega_{\sigma}=\Omega$, i.e. $r=\sin (k y)$. The result is shown in Fig. 3(c). Peaks appear at the antinodes, where $r=1$. By changing the location of the screen, the visibility could be recovered. In Fig. 3(d), we choose the distance $y$ to correspond with the values of $r$ in Fig. 3(b), and we obtain the same results. In other words, when the relative phase is fixed to $\pi / 2$, the interference patten in $x-z$ plane could be revealed by moving the screen along the $y$ direction. Controlling the distance between the atoms and the screen is an alternative choice as compare to adjust the intensities of the driving fields.

In summary, the phase-dependent interference of resonance fluorescence from two duplicated two-level atoms is investigated. The interference patten can be recovered in the fluorescence light of strongly driven atoms due to the relative phase between the two driving fields. However, when $\phi=\pi / 2$, the intensity is zero, and no fluorescence could be detected. By adjusting the relative intensities, this problem can be solved. A scheme of recovering the visibility by using a standing-wave field is proposed too. By replacing the $\pi$-polarized field with a standing wave, the interference patten in $x-z$ plane could by revealed
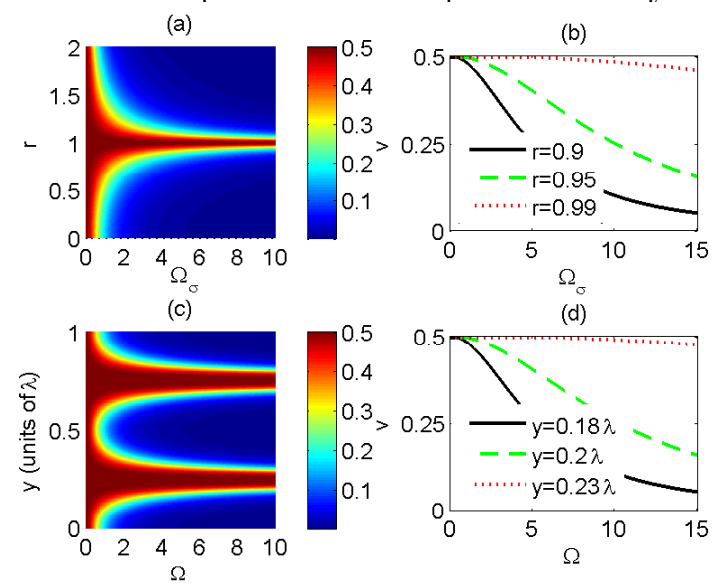

FIG. 3: (Color online) The visibility $V$ when $\phi=\pi / 2$. (a) $V$ as the functions of $r$ and the intensity $\Omega_{\sigma}$. (b) Some examples in (a) when $r$ is adjusted. (c) $V$ as the functions of the position $y$ and the intensity $\Omega$. (d) Some examples in (c).

by moving the screen along the $y$ direction.

This work is supported by the 973 Program under Grant No. 2006CB921104, and the National Natural Sciences Foundation of China (Grant No. 60708008, 60921004, 60978013, 10874194).
[1] M. Scully and M. Zubairy, Quantum optics (CUP, Cambridge, 1997).

[2] U. Eichmann et al., Phys. Rev. Lett. 70, 2359 (1993).

[3] P. Kochan et al., Phys. Rev. Lett. 75, 45 (1995); G. M. Meyer and G. Yeoman, ibid 79, 2650 (1997).

[4] W. H. Itano et al., Phys. Rev. A 57, 4176 (1998); T. Rudolph and Z. Ficek, ibid 58, 748 (1998); G. Yeoman, ibid 58, 764 (1998); Ch. Schön and Almut Beige, ibid 64, 023806 (2001); C. Skornia et al., ibid 64, 063801 (2001); L. Jin et al., Opt. Commun. 283, 790 (2010).

[5] T. Wong et al., Phys. Rev. A 55, 1288 (1997).

[6] G. S. Agarwal et al., Phys. Rev. A 65, 053826 (2002).
[7] V. Shatokhin et al., Phys. Rev. Lett. 94, 043603 (2005).

[8] W. Vogel and D.-G. Welsch, Phys. Rev. Lett. 54, 1802 (1985).

[9] A. N. Boto et al., Phys. Rev. Lett. 85, 2733 (2000); M. D'Angelo, M. V. Chekhova and Y. Shih, ibid. 87, 013602 (2001).

[10] M. Macovei and C. Keitel, Phys. Rev. B 75, 245325 (2007).

[11] M. Macovei et al., Phys. Rev. Lett. 98, 043602 (2007).

[12] F. Hashmi and M. Bouchene, Phys. Rev. Lett. 101, 213601 (2008).

[13] M. S. Shahriar and P. R. Hemmer, Phys. Rev. Lett. 65, 
1865 (1990); W. Maichen et al., Phys. Rev. A 53, 3444

(1996); N. Ph. Georgiades et al., Opt. Lett. 21, 1688

(1996); E. A. Korsunsky et al., Phys. Rev. A 592302 (1999).
[14] D. Polder and M.F.H Schuurmans, Phys. Rev. A 14, 1468 (1976). 